\title{
Denosumab for the prevention of osteoporotic fractures in postmenopausal women
}

\author{
N Waugh, ${ }^{1 *}$ P Royle, ${ }^{1}$ G Scotland, ${ }^{2}$ \\ $\mathrm{R}$ Henderson, ${ }^{3} \mathrm{R}$ Hollick ${ }^{4}$ and $\mathrm{P}$ McNamee ${ }^{2}$
}

${ }^{1}$ Department of Public Health, University of Aberdeen, Aberdeen, UK

${ }^{2}$ Health Economics Research Unit, University of Aberdeen, Aberdeen, UK

${ }^{3} \mathrm{NHS}$ Highland, Inverness, UK

${ }^{4}$ Aberdeen Royal Infirmary, Aberdeen, UK

${ }^{*}$ Corresponding author

Declared competing interests of authors: none

\author{
HTA 08/231/01 \\ Date of ERG submission: \\ 23 March 2010 \\ TAR Centre(s): \\ Aberdeen HTA Group \\ List of authors: \\ N Waugh, P Royle, G Scotland, R Henderson, R Hollick and P McNamee \\ Contact details: \\ Professor NR Waugh, Department of Public Health, Medical School Buildings, Foresterhill, Aberdeen AB 25 \\ 2ZD, UK \\ E-mail: n.r.waugh@abdn.ac.uk \\ The research reported in this article of the journal supplement was commissioned and funded by the HTA \\ programme on behalf of NICE as project number 08/231/01. The assessment report began editorial review \\ in September 2010 and was accepted for publication in October 2010. See the HTA programme website for \\ further project information (www.hta.ac.uk). This summary of the ERG report was compiled after the Appraisal \\ Committee's review. \\ The views and opinions expressed therein are those of the authors and do not necessarily reflect those of the \\ Department of Health. \\ Discussion of ERG reports is invited. Visit the HTA website correspondence forum (www.hta.ac.uk/correspond).
}

\section{Abstract}

This paper presents a summary of the Evidence Review Group (ERG) report into denosumab for the prevention of osteoporotic fractures in postmenopausal women.

Denosumab has been shown in a large randomised trial to reduce the frequency of osteoporotic fractures, when given subcutaneously at 6-monthly intervals. Compared with placebo, the 
relative risks of clinical vertebral and hip fractures were 0.32 and 0.60 , respectively. Clinical vertebral fractures occurred in $0.8 \%$ of women taking denosumab and $2.6 \%$ of control subjects. Hip fractures occurred in $1.2 \%$ of women on placebo and $0.7 \%$ on denosumab. The expected use is in women who cannot tolerate oral bisphosphonates. Other options in that situation include strontium ranelate and zoledronate, which, compared with placebo, also reduced the risk of clinical vertebral fractures [relative risks (RRs) 0.65 and 0.23 , respectively]. Zoledronate also significantly reduced the risk of hip fractures (RR 0.59). The ERG concluded that zoledronate was the main comparator. The relative cost-effectiveness of denosumab and zoledronate depends mainly on assumptions about costs of administration.

\section{Introduction}

The National Institute for Health and Clinical Excellence (NICE) is an independent organisation within the NHS. One of the responsibilities of NICE is to provide guidance to the NHS on the use of selected new and established health technologies, based on an appraisal of those technologies.

NICE's single technology appraisal (STA) process is specifically designed for the appraisal of a single product, device or other technology, with a single indication, where most of the relevant evidence lies with one manufacturer or sponsor. ${ }^{1}$ Typically, it is used for new pharmaceutical products close to launch. The principal evidence for an STA is derived from a submission by the manufacturer/sponsor of the technology. In addition, a report reviewing the evidence submission is submitted by the evidence review group (ERG), an external organisation independent of the Institute. This paper presents a summary of the ERG report for the STA entitled 'Denosumab for the prevention of osteoporotic fractures in postmenopausal women.'

\section{Description of the underlying health problem}

Postmenopausal osteoporosis has been defined as ' ... a progressive, systemic skeletal disorder characterised by low bone mass and micro-architectural deterioration of bone tissue, with a consequent increase in bone fragility and susceptibility to fracture. ${ }^{3}$

It is estimated that there are 180,000 osteoporosis-related symptomatic fractures each year in England and Wales. ${ }^{3}$ Of these, 70,000 are hip fractures that require hospital admission and surgical treatment, and are associated with significant morbidity and mortality. ${ }^{3}$ In women over the age of 50 years, the lifetime risk of a hip fracture is one in five. ${ }^{3} \mathrm{~A}$ recent meta-analysis of all anti-osteoporosis drugs found that treatment reduces mortality. ${ }^{4}$

\section{Scope of the ERG report}

Bone is in a state of continuous breakdown and renewal. Breakdown is carried out by cells called osteoclasts and renewal by osteoblasts. The cytokine RANK-ligand plays a pivotal role in mediating osteoclast activity. Denosumab is a human monoclonal antibody that inhibits RANKligand, thereby reducing osteoclast activity and hence bone breakdown.

Marketing authorization for denosumab has been granted by the Committee for Medicinal Products for Human Use (CHMP) of the European Medicines Agency (EMA). ${ }^{5}$

The manufacturer's submission (MS) argued that denosumab was clinically effective and cost-effective in the prevention of osteoporotic fractures among postmenopausal women. The submission assumed that because of the low cost of oral bisphosphonates (OBPs), denosumab would be used in women who were unable to tolerate those drugs. 
In a large trial against placebo (the FREEDOM trial ${ }^{6}$ ), denosumab was shown to reduce fracture risk. The MS included evidence from studies comparing denosumab against several comparator treatments with changes in bone mineral density (BMD) as the primary end point. However, given that fracture rates have been reported in trials of denosumab and comparator treatments, that evidence was preferred, and information relating to BMD was not considered by the ERG.

\section{Methods}

The ERG report comprised a critical review of the evidence for the clinical effectiveness and cost effectiveness of denosumab presented in the MS to NICE.

Reviews of the clinical effectiveness of treatment for osteoporosis had been conducted ${ }^{7}$ to support the development of a NICE osteoporosis guideline. ${ }^{8}$ The manufacturer updated these by searching for studies published since these reviews. The ERG also conducted searches to identify if any relevant studies had been missed.

The control group in the FREEDOM trial ${ }^{6}$ was given placebo. The effectiveness of denosumab relative to other bone-loss therapies was therefore estimated from an indirect comparison using trials of other drugs against placebo, using the adjusted indirect comparison method described by Bucher and colleagues. ${ }^{9}$ Although not ideal, this is the only approach that could be adopted in the absence of head-to-head trials.

The ERG explored the challenges associated with the adjusted indirect comparison method, and considered whether differences in the baseline characteristics of studies included in the indirect comparison (which could modify the relevant treatment effect) had been taken into account by the manufacturer.

The manufacturer used an economic model that took account of costs from short-term drug costs to long-term nursing home costs. The ERG evaluated the model against the 10-point checklist developed by Drummond and colleagues ${ }^{10}$ and the NICE reference case. ${ }^{11}$ The ERG also sought opinion regarding assumptions made within the manufacturer's model, following which the model was re-run after making adjustments based on the views and information received.

The manufacturer had also conducted a systematic review of economic studies assessing the costs and/or cost-effectiveness of denosumab. The ERG undertook further searches to identify if any relevant studies had been missed.

\section{Results}

\section{Summary of submitted clinical evidence}

The main evidence submitted was the FREEDOM trial of denosumab against placebo, ${ }^{6}$ and an indirect comparison of denosumab against other drugs.

The FREEDOM trial ${ }^{6}$ was a large, good-quality trial involving women with postmenopausal osteoporosis. ${ }^{12}$ Denosumab given by subcutaneous injection at 6-monthly intervals for 3 years was effective in reducing fractures. Hip fractures were reduced by $40 \%$, from $1.2 \%$ of women in the placebo group to $0.7 \%$ in the denosumab group. Clinical vertebral fractures were reduced by $69 \%$ from $2.6 \%$ in the placebo group to $0.8 \%$ in the denosumab group. 
Safety data are available from 30 studies with 14,000 patients, of whom 11,000 are postmenopausal women. Based on these studies, denosumab appears safe. However, a US Food and Drug Administration (FDA) summary of safety ${ }^{13}$ noted that people receiving denosumab appeared to have a slightly increased risk of serious infections of the skin, ear, urinary tract and abdomen. They also noted a non-significant increase in cancer incidence, and theoretical concerns about denosumab suppressing dynamic bone formation leading to delayed fracture healing and atypical fracture. ${ }^{13}$

Persistence with osteoporosis treatment is known to be poor for many existing drugs, such as the OBPs. ${ }^{14-16}$ It is likely that the 6-monthly administration in GP surgery or hospital clinic will encourage persistence with denosumab.

The MS stated that because of the availability of inexpensive generic OBPs ' $\ldots$ denosumab is expected to be an appropriate option for diagnosed patients for whom oral BPs are unsuitable; reasons for unsuitability include inability to comply with the special instructions for administration, a contraindication or intolerance' (p. 15). The drugs used in the indirect comparison were therefore strontium, raloxifene, teriparatide, zoledronate and intravenous (i.v.) ibandronate.

The indirect comparison included a comparison of the relative risks (RRs) of fracture for each drug versus placebo, and an adjusted estimation of the RR of fracture for denosumab versus the other drugs. This demonstrated that denosumab, strontium ranelate and zoledronate provided statistically significant decreases in the risk of clinical vertebral fractures (RRs of $0.32,0.65$ and 0.23 , respectively) compared with placebo, but raloxifene did not ( $R R$ of 0.45 , not significant). Denosumab and zoledronate reduced the risk of hip fractures compared to placebo (RRs of 0.61 and 0.59 respectively, both statistically significant) but strontium ranelate did not (RR 0.89 , not significant). Data on the risk of wrist fractures relative to placebo were available for denosumab (RR 0.84 ) and strontium ranelate (RR 0.98 ), neither of which was statistically significant. No data on wrist fractures were available for zoledronate or raloxifene. The RRs obtained from the direct comparison of each drug with placebo were used to model cost-effectiveness.

\section{Summary of submitted cost-effectiveness evidence}

The manufacturer provided multiple comparisons of cost-effectiveness using a good-quality Markov model that took account of drug costs, administration and monitoring costs, costs associated with fractures, and long-term nursing home costs. Utility weights derived from a review of the literature were used to adjust time spent in fracture states, allowing quality-adjusted life-years (QALYs) to be estimated. The base-case analysis was conducted for 70-year old-women with a $T$-score of $\leq-2.5$ and no prior fracture, and 70-year old-women with a $T$-score of $\leq-2.5$ with a prior fragility fracture. Subgroup analyses based on $T$-score and independent clinical risk factors were also undertaken. The analysis complied with the NICE reference case.

The submission argued that denosumab:

- dominated strontium for both primary and secondary prevention, i.e. was both more effective and less costly

- was cost-effective compared with raloxifene, with costs per QALY of $£ 9289$ in primary prevention and around $£ 2000$ in secondary prevention

- could be cost-effective compared with no treatment in some subgroups of women without prior fracture, who might not be treated with a second drug if unable to tolerate OBPs, according to the current NICE guidance

- could be cost-effective versus no treatment in women with fragility fractures $(\mathfrak{E} 12,381$ per QALY). 
- dominated i.v. ibandronate

- was cost-effective compared with zoledronate with the incremental cost-effectiveness ratio (ICER) for zoledronate versus denosumab reported to be $£ 70,000$ per QALY in women with no prior fracture and $£ 29,000$ in women with a prior fracture.

However, a key assumption was that denosumab would be given in general practice at the average cost of two standard GP visits a year. This would make it less costly than zoledronate, which is given by i.v. infusion, in hospital clinics, once a year. Given the similar effectiveness of denosumab and zoledronate, the cost-effectiveness comparison depended largely on the relative costs. The ERG were of the opinion that GPs would be likely to regard administration of a new biological agent as not part of General Medical Services, and might expect it to be part of an enhanced service. The ERG also expected that denosumab would be initiated in secondary care, and that patients might also be followed up in secondary care.

\section{Commentary on the robustness of submitted evidence Strengths}

The key trials considered were of good quality, had large numbers of recruits and were of adequate duration. The economic model was of high quality. The submission and appendices provided very detailed accounts of underlying assumptions and sensitivity analyses.

\section{Weaknesses}

The manufacturer submitted a very large amount of material, far exceeding the NICE guidance on length of submission. However, the ERG considered the evidence of effects of drugs on bone mineral density to be not relevant, partly because of doubts about the value of BMD in assessing effects of most drugs in osteoporosis, but mainly because there were fracture data for all the drugs. Nor did the ERG consider the data on morphometric vertebral fractures to be useful, and it was noted that Amgen did not use such data in the modelling.

The Amgen submission argued that zoledronate and i.v. ibandronate should not be primary comparators because they were 'not standard care' and because they had not been appraised by NICE. Howeve, despite not having been appraised by NICE, both have been licensed for some time and are in routine use in the UK.

In the ERG's opinion, the major weakness lay in the economic modelling of zoledronate versus denosumab. The ERG considered zoledronate to be a key comparator for denosumab, and the relative cost-effectiveness of these two drugs was sensitive to assumptions about relative costs of administering them.

If the cost of denosumab was increased, by assuming it would be delivered in secondary care, the ICER for denosumab compared with no treatment would rise to $£ 36,185$ per QALY in women with no prior fragility fracture, and to $£ 15,720$ per QALY in women with a prior fragility fracture. This change led to zoledronate dominating denosumab in women with and without a prior fragility fracture.

The ERG also re-ran the model, assuming equal efficacy of denosumab and zoledronate for the prevention of wrist fractures. This reduced the costs per QALY for zoledronate relative to denosumab by about $£ 10,000$ in primary prevention, and by about $£ 5000$ in secondary prevention.

The MS also examined the cost-effectiveness of denosumab in women who could not tolerate OBPs, but whose risk level meant that other drugs were not currently cost-effective, according to NICE Technology Appraisals (TAs) 160 and 161. ${ }^{3,17}$ The ERG considered this data and surmised 
that denosumab might be considered cost-effective in some of these women if the manufacturer's costing assumptions were to be accepted (assuming a cost-effectiveness threshold of $£ 30,000$ per QALY). This could provide a partial solution to the unsatisfactory situation in which clinicians could not offer an alternative treatment to women unable to tolerate OBPs until their clinical condition deteriorated.

Following advice from clinical experts, the NICE Appraisal Committee ' concluded that it is likely that treatment will be started in secondary care and subsequently delivered in primary care, but with follow up of women with severe osteoporosis in secondary care in accordance with current UK clinical practice'.

\section{Conclusions}

The clinical effectiveness of denosumab is not in doubt, and it appears safe. The key issue in the cost-effectiveness analysis is its cost relative to zoledronate. For women with no prior fragility fractures, its potential cost-effectiveness relative to no treatment in some groups is also highly relevant, as current NICE guidance recommends no treatment for many women in this group if they cannot tolerate OBPs.

\section{Areas of uncertainty}

The indirect comparison was necessary because of the lack of direct head-to-head trials. It appeared to be well done, but the ERG wondered if differences in baseline characteristics of the women in the trials (such as duration of follow-up, age, body mass index, proportion with previous fractures) would affect some comparisons.

Because of absence of data on the effect of zoledronate on wrist fractures, the Amgen modelling assumed that it would not reduce the incidence of those, whereas it was assumed that denosumab would, based on data from the FREEDOM trial. ${ }^{6}$ However, given the equivalence, or a nonsignificant slight superiority, of zoledronate to denosumab, the ERG considered it unlikely that zoledronate would have no effect on wrist fractures.

In the modelling, the reduction in breast cancer incidence from raloxifene treatment was not included. This was raised with the manufacturer, who stated that this was in line with the precedent set in NICE TAs 160 and 161.

In the indirect comparison, data from a trial of oral ibandronate were used, and assumed to apply to i.v. ibandronate. However, the DIVA trial ${ }^{18}$ of oral versus i.v. ibandronate showed that the i.v. form, given at 3-monthly intervals, was more effective, with fracture incidence of $4.8 \%$ in the i.v. groups versus $6.2 \%$ in the oral group. This difference was at 2 years' follow-up and was not statistically significant, but it could be used in a sensitivity analysis.

Two recent studies using data from British general practice have examined the risk of oesophageal cancer. The first, by Cardwell and colleagues, ${ }^{19}$ was a case-control study that started with OBP use, and compared oesophageal cancer rates in users versus age- and sex-matched control subjects who did not take OBPs. They found no increase in cancer risk [RR 1.07, 95\% confidence interval (CI) 0.77 to 1.49]. The second, by Green and colleagues started with cancer cases, and compared OBP use in cancer cases and non-cancer controls. Green and colleagues, ${ }^{20}$ concluded that the risk was increased in patients taking OBPs (RR 1.30, 95\% CI 1.02 to 1.66). The difference may be explained by longer duration of OBPs use in the Green study, ${ }^{20}$ and a subgroup in the Cardwell study ${ }^{19}$ with longer use also has an increase (RR $1.23,95 \% \mathrm{CI}-0.66$ to 0.2 .30 ). 
Summary of NICE guidance (from FAD, as issued 8 September 2010)

1.1 Denosumab is recommended as a treatment option for the primary prevention of osteoporotic fragility fractures only in postmenopausal women at increased risk of fractures:

- who are unable to comply with the special instructions for the administration of OBPs, are intolerant of OBPs or for whom treatment with OBPs is contraindicated and

- who also have a combination of $T$-score, age and number of independent clinical risk factors for fracture (see section 1.3) as indicated in the following table.

\begin{tabular}{llll}
\hline \multicolumn{4}{l}{-scores (SD) at (or below) which denosumab is recommended when OBPs are unsuitable } \\
\hline \multicolumn{4}{l}{ No. of independent clinical risk factors for fracture } \\
\cline { 2 - 4 } Age (years) & $\mathbf{0}$ & $\mathbf{1}$ & $\mathbf{2}$ \\
\hline $65-69$ & $-\mathrm{a}$ & -4.5 & -4.0 \\
$70-74$ & -4.5 & -4.0 & -3.5 \\
75 or older & -4.0 & -4.0 & -3.0
\end{tabular}

OBP, oral bisphosphonate; $\mathrm{SD}$, standard deviation.

a Treatment with denosumab is not recommended.

1.2 Denosumab is recommended as a treatment option for the secondary prevention of osteoporotic fragility fractures only in postmenopausal women at increased risk of fractures who are unable to comply with the special instructions for the administering alendronate and either risedronate or etidronate, or have an intolerance of, or a contraindication to, those treatments.

1.3 For the purposes of this guidance, independent clinical risk factors for fracture are parental history of hip fracture, alcohol intake of four or more units per day, and rheumatoid arthritis.

1.4 People currently receiving denosumab for the primary or secondary prevention of osteoporotic fragility fractures who do not meet the criteria specified in recommendations 1.1 or 1.2 should have the option to continue treatment until they and their clinician consider it appropriate to stop.

\section{Key references}

1. National Institute for Health and Clinical Excellence (NICE). Guide to the single technology appraisal process. October 2009. URL: www.nice.org.uk/media/913/06/Guide_to_the_STAproof_6-26-10-09.pdf (accessed 7 September 2010).

2. Scotland G, Royle P, Henderson R, Hollick R, McNamee P, Waugh N. Denosumab for the prevention of osteoporotic fractures in post-menopausal women: a single technology appraisal. Aberdeen HTA Group, 2010.

3. National Institute for Health and Clinical Excellence (NICE). TA160 Osteoporosis - primary prevention: guidance. January 2010. URL: guidance.nice.org.uk/TA160/Guidance/pdf/English (accessed 17 March 2010).

4. Bolland MJ, Grey AB, Gamble GD, Reid IR. Effect of osteoporosis treatment on mortality: a meta-analysis. J Clin Endocrinol Metab 2010;95:1174-81. 
5. European Medicines Agency (EMA). Prolia. June 2010. URL: www.ema.europa.eu/ ema/index.jsp?curl=pages/medicines/human/medicines/001120/human_med_001324. jsp\&murl $=$ menus $/$ medicines $/$ medicines.jsp\&mid $=$ WC0b01ac058001d $124 \&$ jsenabled $=$ true (accessed 7 September 2010).

6. Cummings SR, San MJ, McClung MR, Siris ES, Eastell R, Reid IR, et al. Denosumab for prevention of fractures in postmenopausal women with osteoporosis. N Engl J Med 2009;361:756-65.

7. National Collaborating Centre for Nursing and Supportive Care. Osteoporosis: evidence review. September 2008. URL: www.nice.org.uk/guidance/index.jsp?action=folder \&o=42361 (accessed 6 February 2010).

8. National Institute for Health and Clinical Excellence (NICE). Osteoporosis. June 2009. URL: http://guidance.nice.org.uk/CG/Wave7/32 (accessed 7 September 2010).

9. Bucher HC, Guyatt GH, Griffith LE, Walter SD. The results of direct and indirect treatment comparisons in meta-analysis of randomized controlled trials. J Clin Epidemiol 1997;50:683-91.

10. Drummond M, Manca A, Sculpher M. Increasing the generalizability of economic evaluations: recommendations for the design, analysis, and reporting of studies. Int J Technol Assess Health Care 2005;21:165-71.

11. National Institute for Health and Clinical Excellence (NICE). Guide to the Methods of Technology Appraisal. April 2004. URL: www.nice.org.uk/media/D15/34/TAP.pdf (accessed 7 September 2010).

12. Black DM, Delmas PD, Eastell R, Reid IR, Boonen S, Cauley JA, et al. Once-yearly zoledronic acid for treatment of postmenopausal osteoporosis. N Engl J Med 2007;356:1809-22.

13. US Food and Drug Administration (FDA). Background document for meeting of advisory committee for reproductive health drugs. 13/11/2009. 13 August 2009. URL: www.fda.gov/downloads/AdvisoryCommittees/CommitteesMeetingMaterials/Drugs/ ReproductiveHealthDrugsAdvisoryCommittee/UCM176605.pdf (accessed 17 March 2010).

14. Boonen S, Vanderschueren D, Venken K, Milisen K, Delforge M, Haentjens P. Recent developments in the management of postmenopausal osteoporosis with bisphosphonates: enhanced efficacy by enhanced compliance. J Intern Med 2008;264:315-32.

15. Carmona R, Adachi R. Treatment of postmenopausal osteoporosis, patient perspectives: focus on once yearly zoledronic acid. Patient Prefer Adherence 2009;3:189-93.

16. Cramer JA, Gold DT, Silverman SL, Lewiecki EM. A systematic review of persistence and compliance with bisphosphonates for osteoporosis. Osteoporos Int 2007;18:1023-31.

17. National Institute for Health and Clinical Excellence (NICE). TA161 Osteoporosis - secondary prevention including strontium ranelate: guidance. January, 2010. http://guidance.nice.org.uk/ TA161/Guidance/pdf/English (accessed 17 March 2010).

18. Eisman JA, Civitelli R, Adami S, Czerwinski E, Recknor C, Prince R, et al. Efficacy and tolerability of intravenous ibandronate injections in postmenopausal osteoporosis: 2-year results from the DIVA study. J Rheumatol 2008;35:488-97.

19. Cardwell CR, Abnet CC, Cantwell MM, Murray LJ. Exposure to oral bisphosphonates and risk of esophageal cancer. JAMA 2010;304:657-63.

20. Green J, Czanner G, Reeves G, Watson J, Wise L, Beral V. Oral bisphosphonates and risk of cancer of oesophagus, stomach, and colorectum: case-control analysis within a UK primary care cohort. BMJ 2010;341:c4444. 